\title{
High intake of fatty fish, but not of lean fish, affects serum concentrations of TAG and HDL-cholesterol in healthy, normal-weight adults: a randomised trial
}

\author{
Ingrid V. Hagen ${ }^{1}$, Anita Helland ${ }^{1}$, Marianne Bratlie ${ }^{1}$, Karl A. Brokstad ${ }^{2}$, Grethe Rosenlund ${ }^{3}$, \\ Harald Sveier ${ }^{4}$, Gunnar Mellgren ${ }^{5,6}$ and Oddrun A. Gudbrandsen ${ }^{1 *}$ \\ ${ }^{1}$ Department of Clinical Medicine, University of Bergen, Haukeland University Hospital, 5021 Bergen, Norway \\ ${ }^{2}$ Broegelmann Research Laboratory, Department of Clinical Science, University of Bergen, Haukeland University Hospital, \\ 5021 Bergen, Norway \\ ${ }^{3}$ Skretting Aquaculture Research Centre AS, PO Box 48, 4001 Stavanger, Norway \\ ${ }^{4}$ Lerøy Seafood Group ASA, PO Box 7600, 5020 Bergen, Norway \\ ${ }^{5}$ Department of Clinical Science, KG Jebsen Center for Diabetes Research, University of Bergen, Haukeland University Hospital, \\ 5021 Bergen, Norway \\ ${ }^{6}$ Hormone Laboratory, Haukeland University Hospital, 5021 Bergen, Norway
}

(Submitted 9 October 2015 - Final revision received 18 May 2016 - Accepted 2 June 2016 - First published online 1 July 2016)

\section{Abstract}

The aim of the present study was to examine whether high intake of lean or fatty fish (cod and farmed salmon, respectively) by healthy, normal-weight adults would affect risk factors of type 2 diabetes and CVD when compared with lean meat (chicken). More knowledge is needed concerning the potential health effects of high fish intake ( $>300 \mathrm{~g} /$ week) in normal-weight adults. In this randomised clinical trial, thirty-eight young, healthy, normal-weight participants consumed $750 \mathrm{~g} /$ week of lean or fatty fish or lean meat (as control) for $4 \mathrm{keeks}$ at dinner according to provided recipes to ensure similar ways of preparations and choices of side dishes between the groups. Energy and macronutrient intakes at baseline and end point were similar in all groups, and there were no changes in energy and macronutrient intakes within any of the groups during the course of the study. High intake of fatty fish, but not lean fish, significantly reduced TAG and increased HDL-cholesterol concentrations in fasting serum when compared with lean meat intake. When compared with lean fish intake, fatty fish intake increased serum HDL-cholesterol. No differences were observed between lean fish, fatty fish and lean meat groups regarding fasting and postprandial glucose regulation. These findings suggest that high intake of fatty fish, but not of lean fish, could beneficially affect serum concentrations of TAG and HDL-cholesterol, which are CVD risk factors, in healthy, normal-weight adults, when compared with high intake of lean meat.

\section{Key words: Diets: Salmon: Cod: Chicken: Glucose}

Intake of fish has been reported to beneficially influence body weight, blood lipids and glucose homoeostasis, which can protect against type 2 diabetes and $\mathrm{CVD}^{(1-5)}$. The health benefits of fish consumption have traditionally been attributed mainly to the effect of $n-3$ fatty acids, and, although the TAG-lowering effect of fish oil and long-chain $n-3$ fatty acids is well documented, there is controversy regarding the cholesterol-regulating as well as glucose-regulating effects of marine $n-3$ fatty acids ${ }^{(6-14)}$. Studies suggests that there are other components in fish such as fish proteins that may be beneficial to human health and affect risk factors leading to CVD, beyond $n-3$ fatty acids ${ }^{(15-18)}$. This is supported by results from animal studies showing that fish proteins may have a lowering effect on TAG and cholesterol as well as improve glucose tolerance ${ }^{(19-26)}$. In line with this, we have recently shown that supplementation with cod protein as tablets reduced fasting glucose and LDL-cholesterol as well as postprandial glucose concentrations in healthy, overweight adults ${ }^{(17)}$.

The present study was designed to investigate the impacts of high intake of lean or fatty fish (cod and salmon, respectively) compared with lean meat (chicken) on indicators of CVD and type 2 diabetes risk factors, including lipid and glucose regulation. Serum fructosamine concentration as a measure of the mean glucose concentration over the last $1-3$ weeks ${ }^{(27)}$ was examined. In addition, methylglyoxal (MG) and $N^{\varepsilon}$-(carboxymethyl) lysine (CML) were analysed, as changes in lipid and glucose regulation may affect the production of MG from the glycation process by degradation of glucose, Schiff's bases or from Amadori products such as fructosamine, and the production of $\mathrm{CML}^{(28)}$. Several intervention studies with fish intake have been conducted by others in overweight, dyslipidaemic, hypertensive,

Abbreviations: MG, methylglyoxal; CML, $\mathrm{N}^{\varepsilon}$-(carboxymethyl) lysine; CRP, C-reactive protein.

* Corresponding author: O. A. Gudbrandsen, fax +475597 5890, email oddrun.gudbrandsen@k1.uib.no 
insulin-resistant and/or diabetic subjects ${ }^{(12-13,17-18,29-37)}$; however, as no studies on the effect of high fish intake in a group of young, healthy, non-smoking, normal-weight adults have been published, little is known about how fish intake may affect lipid and glucose metabolism in this population. As the choice of preparation methods of fish and meat as well as the choices of side dishes and accessories may explain some of the different health effects from fish consumption compared with meat ${ }^{(38-41)}$, participants in the present study were instructed to follow a recipe booklet to prepare fish and lean meat dinners, respectively, with comparable preparation methods and side dishes. In the present study, we wanted to test our hypothesis that a high intake of lean or fatty fish would not affect circulating concentrations of lipids or glucose tolerance in young, healthy, normal-weight adults with serum concentrations of lipids, glucose and insulin within normal reference ranges, when compared with lean meat intake.

\section{Methods}

\section{Participants, study setting and ethics}

Participants were 1st- to 4th-year students at the Faculty of Medicine and Dentistry, University of Bergen, Norway. The students were invited by members of the research team to participate in the study by using the University's shared email system for students from April to May 2011. Inclusion criteria were normal body weight (BMI $18.5-24.9 \mathrm{~kg} / \mathrm{m}^{2}$ ), fasting blood glucose $\leq 7.0 \mathrm{mmol} / 1$ and $20-35$ years of age. Participants would have to be willing to consume the assigned amount of fish or chicken during the intervention. Exclusion criteria were pregnancy, incompatibility with fish or chicken consumption (allergies, intolerance and/or dislike), diagnosed diabetes mellitus, heart disease or gastrointestinal diseases, use of medications affecting lipid metabolism or glucose homoeostasis, use of anti-inflammatory medications, use of supplements containing long-chain $n$ - 3 fatty acids, intentional weight loss and large fluctuation in body weight $(>3 \mathrm{~kg}$ ) over the previous 2 months, and smoking.

The study was designed as a randomised intervention study with a parallel group design, with three intervention arms: lean fish (cod), fatty fish (salmon) or lean meat (chicken) as the control group in weekly doses of $750 \mathrm{~g}$ (five meals of $150 \mathrm{~g}$ ) for 4 weeks. A total of forty-five students were included in the study and were randomly assigned to lean fish ( $n$ 14), fatty fish ( $n$ 15) or lean meat ( $n$ 16) groups. The participants were stratified into the different intervention groups by the project manager on the basis of sex, age and BMI. All examinations were conducted at the Haukeland University Hospital, Bergen, Norway.

This study was conducted according to the guidelines laid down in the Declaration of Helsinki, and all procedures involving participants were approved by the Regional Committee for Medical and Health Research Ethics (approval no.: 2011/572). Written informed consent was obtained from all subjects. Health professionals performing blood sampling and measuring height and body weight and personnel conducting the laboratory analyses were blinded. All the data were analysed anonymously. This trial was registered at clinicaltrials.gov as NCT02130908.

\section{Interventions}

The participants were instructed to eat 5 meals/week containing $150 \mathrm{~g}$ of cod, salmon or chicken over a period of 4 weeks, and they were told not to exceed the total amount of $750 \mathrm{~g}$ of fish or chicken/week. If $150 \mathrm{~g}$ of fish or chicken/d was not sufficient, participants were encouraged to supplement their meal with vegetables, pasta or rice. Participants in the fish-eating groups were instructed not to consume any other fish or seafood during the study period, and participants in the lean meat group were instructed not to consume fish or seafood during the intervention period. All participants were allowed to eat meat, but not along with the meal in which they consumed fish or meat for the study. The participants were instructed to maintain their normal eating habits throughout the study period, apart from eating the mandatory amount of $750 \mathrm{~g}$ fish or chicken/week, and not to change their physical activity level during the 4-week intervention period. Use of dietary supplements was not allowed during the study period. Participants were instructed to follow a recipe booklet to prepare fish and lean meat dinners, respectively, and to report recipes that they chose for the study meals to see which methods of preparation and accessories the participants preferred and also as a mean to assess compliance. Dietary intakes before start of the study and before end of the study were assessed using 5-d food records at both time points.

Fish was provided as frozen skin and boneless fillet portions (150 (SD 10) g; Lerøy Seafood Group ASA). Chicken fillets (150 (SD 10) g; 'Den stolte hane') were frozen before use in the intervention study. The fish and chicken fillets were supplied free of charge to the participants, and were distributed at baseline or at any time during the study period if preferred. The compositions of the fillets were as follows, presented as mean values and standard deviations for analyses of five samples: proteins (wt \%), cod 20.6 (sD 0.5), salmon 20.0 (sD 0.7) and chicken 24.0 (SD 0.5); moisture (wt\%), cod 77.4 (sD 0.4), salmon 67.0 (sD 2.0) and chicken 72.6 (sD 0.5); and total fats (wt\%), cod 0.67 (SD 0.03), salmon 11.2 (SD 2.2) and chicken 1.7 (SD 0.3). The total content of $\mathrm{EPA}+\mathrm{DHA}$ was 0.33 (SD 0.02), 1.4 (SD 0.2) and $0.02(\mathrm{SD} 0.01) \mathrm{g} / 100 \mathrm{~g}$ filet of cod, salmon and chicken, respectively. The fillets were analysed by skretting ARC Laboratory using standard laboratory methods. Protein content (as Nx6.25) was analysed using the Kjeldahl method ${ }^{(42)}$; moisture was analysed gravimetrically after drying to constant weight in an oven at $105^{\circ} \mathrm{C}^{(43)}$; and fatty acid composition was determined after methylation of the fatty acids in methanolic $\mathrm{HCl}$ and extraction in hexane ${ }^{(44)}$. The methyl esters were separated by gas chromatography (Thermo Trace GC with Triplus autosampler; Thermo Scientific) and identified by retention time using standard mixtures of methyl esters (Nu-Chek), using C19:0 as the internal standard for quantification. Total fat content was calculated as the sum of fatty acids.

\section{Protocol for study visits}

The total study period was 4 weeks, with study visits at baseline and at the end of the study ( 4 weeks). Examinations were conducted in the morning after an overnight fast. The subjects were instructed not to eat or drink anything except water, not to 
use substances containing nicotine after 22.00 hours the previous day and to avoid physical exercise and alcohol for $24 \mathrm{~h}$ before each sampling day.

Body height was measured at baseline, whereas body weight was measured at baseline and at the end of the study. Blood samples were collected at baseline and at the end point in a fasting state. Blood samples were collected in BD Vacutainer SST II Advance gel tubes (Becton, Dickinson and Company) for isolation of serum and Vacuette K2EDTA tubes (Greiner Bio-one) for isolation of plasma.

After collection of fasting blood samples, glucose tolerance was tested using a standardised breakfast meal containing fat and protein in addition to carbohydrates. A standardised meal test was chosen instead of the traditional oral glucose tolerance test, where the subject is given a measured dose (usually $75 \mathrm{~g}$ ) of glucose, as the former gives a more physiological description of the body's response to an oral carbohydrate load ${ }^{(45)}$. The breakfast consisted of two slices $(65 \mathrm{~g})$ of white bread, $7 \cdot 5 \mathrm{~g}$ margarine, $50 \mathrm{~g}$ strawberry jam and 0.25 litres orange juice, providing a total of $1908 \mathrm{~kJ}$ ( $85 \mathrm{~g}$ carbohydrate, $8 \mathrm{~g}$ protein and $8 \mathrm{~g}$ fat) and had to be consumed within $15 \mathrm{~min}$. The macronutrient and energy contents in the breakfast were calculated using 'Mat på Data 5.1' (www.matportalen.no/ Emner/matpadata) ${ }^{(46)}$. A second blood sample was collected $120 \mathrm{~min}$ after the standardised breakfast. Food records were checked for completeness at both study visits.

\section{Description of recipes}

A detailed description of the recipes can be found in the Online Supplementary File 1. The energy and macronutrient contents in the recipes are presented in Table 1.

\section{Estimation of energy and macronutrient intakes from dietary records}

Participants completed a dietary record of their food intakes of the 5 preceding days before baseline and the 4 -week visit, including at least 1 weekend day. The intakes of energy, carbohydrates, proteins and fats were calculated from the participants' dietary record using 'Mat på Data 5.1' software ${ }^{(46)}$.

\section{Analysis of serum and plasma samples}

Analyses of lipids, glucose, total bile acids, C-reactive protein (CRP), insulin and insulin C-peptide in blood serum were performed by routine methods at the Laboratory of Clinical Biochemistry and the Hormone Laboratory at Haukeland University Hospital, and reference values were set according to these laboratories. Serum NEFA and plasma fructosamine were analysed with the Cobas c111 system (Roche Diagnostics $\mathrm{GmbH}$ ) using the NEFA FS kit (DiaSys; Diagnostic Systems $\mathrm{GmbH}$ ) and the Fructosamine kit (FRA) for Cobas c systems (Roche), respectively. Adiponectin (HADK1MAG-61K; EMD Millipore) was measured in serum using $\mathrm{xMAG}^{\circledR}$, a bead-based multiplex technique (Luminex Corp.) on a Luminex 100 instrument (Luminex Corp.) using STarStation version 3 software (AppliedCytometry). Plasma concentrations of MG and CML were measured using OxiSelect ${ }^{\mathrm{TM}}$ competitive ELISA kits (Cell Biolabs, Inc.) on Spectra Max Plus 384 (Molecular devices LLC), with readings at $450 \mathrm{~nm}$.

\section{Outcome measurements}

The primary outcome of the present study was changes in serum concentrations of TAG after a weekly intake of 750-g fillet of either lean or fatty fish, or lean meat. Secondary outcomes were changes in cholesterols, glucose, insulin, insulin C-peptide, body weight and intakes of energy and macronutrients within the groups over time, as well as a comparison of choices of preparation methods, side dishes and accessories between the groups.

\section{Statistical analyses}

We consider the present study to be a pilot study, as to our knowledge this is the first study on the effects of high fish intake compared with lean meat intake in a group of young healthy, non-smoking normal-weight adults with serum concentrations of lipids, glucose and insulin within normal reference ranges. Other studies of fish intake in other populations have shown that a sample size of between ten and fifteen was sufficient to observe a lowering effect of fish intake on serum TAG concentration $^{(29,31,33)}$. Therefore, as there is no information available about the necessary sample size, we consider the present study to be hypothesis generating rather than hypothesis testing, and the study will constitute a base for sample size calculations for future studies with similar designs.

Statistical analyses were conducted using PASW Statistics version 22 (SPSS, Inc., IBM Company). Subjects who did not complete the study were excluded from the statistical analyses. For analytes in serum and estimated energy and macronutrient intakes from dietary records, most data were not normally distributed according to the Shapiro-Wilk test, and nonparametric tests were used to investigate changes within groups (Wilcoxon's signed-rank test). For these non-parametric data, the Kruskal-Wallis test was used to compare values between the three groups at baseline. Changes within the groups were compared using the Kruskal-Wallis test followed by the Mann-Whitney test whenever group differences were detected. Data are expressed as medians and 25th-75th percentiles, with the exception of macronutrient contents in the recipes where the Shapiro-Wilk test revealed that data were normally distributed. Therefore, comparisons of the choices of recipes were conducted using one-way ANOVA, and the results are presented as mean values and standard deviations. All comparisons were two-sided, and $P<0.05$ was considered to be statistically significant.

\section{Results}

\section{Participant characteristics}

A total of forty-five students were included in the study and were randomly assigned to one of the three experimental 
groups eating five dinner meals per week containing $150 \mathrm{~g}$ of either lean fish (cod, $n 14$ ), fatty fish (salmon, $n 15$ ) or lean meat (chicken, $n$ 16). There were two dropouts (both from the lean meat group), and five participants were withdrawn from analysis because they did not comply with the protocol or because of onset of the use of dietary supplements or medications affecting glucose and/or lipid metabolism during the study period. For this reason, the statistical analysis included thirty-eight participants (fifteen men and twenty-three women) who completed the 4-week intervention and fulfilled the criteria (lean fish, $n 13$ (five men); fatty fish, $n 14$ (six men); lean meat, $n 11$ (four men)). All participants were young and apparently healthy adults with normal BMI. The median age was 23.2 (25th-75th percentile $20.9,24.8$ ) years, and the median BMI was $21 \cdot 1$ (25th-75th percentile $19 \cdot 5,22 \cdot 6) \mathrm{kg} / \mathrm{m}^{2}$. Serum concentrations of lipids, glucose and insulin were within normal reference ranges at baseline. No statistically significant differences were found between the intervention groups at baseline for age (lean fish, 23.2 (25th-75th percentile 21.6, 24.6); fatty fish, 24.5 (25th-75th percentile 22.8, 24.9); lean meat, 20.7 (25th-75th percentile $20.5,26 \cdot 0$ ) years) or BMI (lean fish, 21.2 (25th-75th percentile 18.8, 22.8); fatty fish, 20.8 (25th-75th percentile 19.9, 22.1); lean meat, 21.2 (25th-75th percentile $19 \cdot 7,23 \cdot 5) \mathrm{kg} / \mathrm{m}^{2}$ ). BMI was not affected by 4 weeks of fish intake (data not presented).

\section{Estimated dietary intake}

The participants registered their choices of recipes from the provided booklets during the 4 -week intervention period. The preferences for preparation methods and side dishes showed considerable variation within each intervention group, but no statistically significant differences were seen between the three intervention groups regarding choice of recipes (Table 2). As presented in Table 1, the estimated energy contents were comparable between Recipes 1-5 and were noticeably higher

Table 1. Estimated energy and macronutrient content in the recipes from the provided booklets

\begin{tabular}{|c|c|c|c|c|c|c|c|}
\hline & Energy (kJ) & Protein $(\mathrm{g})$ & $\mathrm{CH}(\mathrm{g})$ & Total fat $(\mathrm{g})$ & SFA (g) & MUFA (g) & PUFA (g) \\
\hline \multicolumn{8}{|l|}{ Recipe 1} \\
\hline Lean fish & 1360 & 37.9 & 29.0 & $5 \cdot 3$ & 2.9 & 1.4 & 1.0 \\
\hline Fatty fish & 2130 & 37.0 & 29.0 & 23.8 & 5.4 & $9 \cdot 1$ & $6 \cdot 2$ \\
\hline Lean meat & 1983 & 43.2 & 29.7 & $18 \cdot 4$ & 4.2 & 8.6 & 4.4 \\
\hline \multicolumn{8}{|l|}{ Recipe 2} \\
\hline Lean fish & 1983 & $42 \cdot 7$ & 34.6 & $18 \cdot 3$ & $5 \cdot 8$ & $10 \cdot 0$ & 1.8 \\
\hline Fatty fish & 2757 & 41.8 & 34.6 & $36 \cdot 8$ & 8.2 & $17 \cdot 8$ & 7.0 \\
\hline Lean meat & 2192 & $47 \cdot 7$ & 34.6 & 20.5 & $6 \cdot 3$ & $10 \cdot 7$ & $2 \cdot 1$ \\
\hline \multicolumn{8}{|l|}{ Recipe 3} \\
\hline Lean fish & 2109 & $42 \cdot 7$ & 54.0 & $12 \cdot 2$ & 1.9 & 8.4 & 1.8 \\
\hline Fatty fish & 2879 & $41 \cdot 8$ & 54.0 & $30 \cdot 7$ & 4.4 & $16 \cdot 2$ & $7 \cdot 0$ \\
\hline Lean meat & 2318 & $47 \cdot 7$ & 54.0 & 14.5 & $2 \cdot 4$ & $9 \cdot 0$ & $2 \cdot 1$ \\
\hline \multicolumn{8}{|l|}{ Recipe 4} \\
\hline Lean fish & 2075 & $42 \cdot 5$ & 38.4 & 19.4 & 3.6 & $10 \cdot 3$ & 4.7 \\
\hline Fatty fish & 2841 & 41.6 & 38.4 & 37.8 & $6 \cdot 1$ & $18 \cdot 0$ & 9.9 \\
\hline Lean meat & 2276 & 47.5 & 38.4 & 21.6 & $4 \cdot 1$ & 11.0 & $5 \cdot 1$ \\
\hline \multicolumn{8}{|l|}{ Recipe 5} \\
\hline Lean fish & 2481 & 43.6 & $34 \cdot 3$ & $32 \cdot 0$ & $13 \cdot 2$ & 13.5 & 3.4 \\
\hline Fatty fish & 3255 & $42 \cdot 7$ & $34 \cdot 3$ & 50.4 & $15 \cdot 7$ & $21 \cdot 2$ & 8.6 \\
\hline Lean meat & 2690 & $48 \cdot 6$ & $34 \cdot 3$ & 34.2 & 13.7 & $14 \cdot 2$ & 3.7 \\
\hline \multicolumn{8}{|l|}{ Recipe 6} \\
\hline Lean fish & 5063 & $64 \cdot 2$ & $110 \cdot 7$ & 53.9 & $20 \cdot 3$ & 24.0 & 5.5 \\
\hline Fatty fish & 5837 & $63 \cdot 3$ & $110 \cdot 7$ & $72 \cdot 3$ & $22 \cdot 8$ & $31 \cdot 7$ & $10 \cdot 8$ \\
\hline Lean meat & 5243 & 69.0 & 109.5 & $56 \cdot 0$ & $20 \cdot 8$ & 24.7 & 5.9 \\
\hline
\end{tabular}

$\mathrm{CH}$, carbohydrates.

Table 2. Choices of recipes for all participants and for the individual intervention groups, shown as percentage of all choices* (Mean values and standard deviations)

\begin{tabular}{|c|c|c|c|c|c|c|c|c|c|}
\hline & \multicolumn{2}{|c|}{ All participants } & \multicolumn{2}{|c|}{ Lean fish } & \multicolumn{2}{|c|}{ Fatty fish } & \multicolumn{2}{|c|}{ Lean meat } & \multirow[b]{2}{*}{$P \dagger$} \\
\hline & Mean & SD & Mean & SD & Mean & SD & Mean & SD & \\
\hline Recipe 1: baked fish/meat with root vegetables & 20 & 11 & 18 & 12 & 21 & 9 & 21 & 12 & 0.87 \\
\hline Recipe 2: roasted fish/meat with potatoes and broccoli & 19 & 13 & 11 & 9 & 24 & 15 & 21 & 11 & 0.14 \\
\hline Recipe 3: wok with fish/meat & 16 & 11 & 21 & 11 & 15 & 9 & 12 & 9 & 0.069 \\
\hline Recipe 4: baked fish/meat with pasta & 17 & 12 & 15 & 11 & 15 & 12 & 21 & 12 & 0.35 \\
\hline Recipe 5: fish/meat burger & 15 & 12 & 13 & 10 & 13 & 15 & 19 & 10 & 0.40 \\
\hline Recipe 6: taco with fish/meat & 13 & 13 & 21 & 11 & 13 & 14 & 7 & 8 & 0.67 \\
\hline
\end{tabular}

* Results are presented for thirteen participants in the lean fish group, fourteen participants in the fatty fish group and eleven participants in the lean meat group. $\dagger P$ values between groups. Between-group changes were tested using one-way ANOVA. 
Table 3. Estimated daily dietary intakes of energy and macronutrients (as percentage of energy intake) based on 5-d dietary records at baseline and after 4 weeks*

(Medians and 25th-75th percentiles)

\begin{tabular}{|c|c|c|c|c|c|c|}
\hline & \multicolumn{2}{|c|}{ Baseline } & \multicolumn{2}{|c|}{4 weeks } & \multirow[b]{2}{*}{$P+$} & \multirow[b]{2}{*}{$P \neq$} \\
\hline & Median & 25th-75th percentile & Median & 25th-75th percentile & & \\
\hline \multicolumn{7}{|l|}{ Energy (kJ/d) } \\
\hline Lean fish & 8205 & 7075,11050 & 7682 & 6766,10071 & 0.55 & \multirow[t]{3}{*}{0.34} \\
\hline Fatty fish & 8970 & 7401,12380 & 10012 & 8540,11883 & 0.73 & \\
\hline Lean meat & 7418 & 6523,9699 & 7201 & 6740,11636 & 0.29 & \\
\hline \multicolumn{7}{|c|}{ Protein (\% energy) } \\
\hline Lean fish & 18 & 13,22 & 19 & 16,22 & 0.15 & \multirow[t]{3}{*}{0.63} \\
\hline Fatty fish & 17 & 15,18 & 18 & 16,21 & 0.096 & \\
\hline Lean meat & 17 & 15,20 & 22 & 17,24 & 0.093 & \\
\hline \multicolumn{7}{|c|}{ Total fat (\% energy) } \\
\hline Lean fish & 35 & 27,48 & 32 & 23,42 & 0.60 & \multirow[t]{3}{*}{0.36} \\
\hline Fatty fish & 32 & 29,38 & 37 & 33,46 & 0.18 & \\
\hline Lean meat & 36 & 28,43 & 37 & 27,50 & 0.65 & \\
\hline \multicolumn{7}{|c|}{ SFA (\% energy) } \\
\hline Lean fish & 13 & 11,22 & 11 & 9,17 & 0.70 & \multirow{3}{*}{0.45} \\
\hline Fatty fish & 13 & 11,14 & 14 & 11,16 & 0.36 & \\
\hline Lean meat & 13 & 10,15 & 13 & 10,20 & 0.39 & \\
\hline \multicolumn{7}{|c|}{ cis-MUFA (\% energy) } \\
\hline Lean fish & 12 & 9,16 & 11 & 8,16 & 0.75 & \multirow[t]{3}{*}{0.21} \\
\hline Fatty fish & 10 & 9,11 & 12 & 10,15 & 0.064 & \\
\hline Lean meat & 12 & 10,15 & 13 & 9,17 & 0.80 & \\
\hline \multicolumn{7}{|c|}{ cis-PUFA (\% energy) } \\
\hline Lean fish & 5 & 4,8 & 5 & 3,9 & 0.75 & \multirow[t]{3}{*}{0.55} \\
\hline Fatty fish & 6 & 5,8 & 7 & 5,12 & 0.22 & \\
\hline Lean meat & 7 & 4,8 & 6 & 5,9 & 0.88 & \\
\hline \multicolumn{7}{|c|}{ Carbohydrates (\% energy) } \\
\hline Lean fish & 43 & 33,48 & 43 & 26,48 & 0.97 & \multirow{3}{*}{0.64} \\
\hline Fatty fish & 46 & 42,48 & 41 & 32,48 & 0.47 & \\
\hline Lean meat & 41 & 32,45 & 39 & 32,51 & 0.72 & \\
\hline
\end{tabular}

* No differences were observed between the groups at baseline (Kruskal-Wallis test). Results are presented for thirteen participants in the lean fish group, fourteen participants in the fatty fish group and eleven participants in the lean meat group.

$\dagger$ Within-group changes are tested using the Wilcoxon's signed-rank test.

‡ Changes within lean fish, fatty fish and lean meat groups are compared using the Kruskal-Wallis test.

in Recipe 6. Owing to the high fat content in fatty fish, the energy and fat contents (especially regarding PUFA) were higher in the recipes for fatty fish when compared with those for lean fish and lean meat.

Participants completed a 5-d dietary record chart before baseline and end point. No differences regarding energy and macronutrient intakes were observed between the groups at baseline or after 4 weeks, and there were no changes within any of the groups during the course of the study (Table 3).

\section{Serum lipids}

Fatty fish intake for 4 weeks reduced the concentration of TAG and increased the concentration of HDL-cholesterol in serum when compared with the lean meat group ( $P$ values of 0.018 and 0.025 , respectively, Table 4$)$. The increase in HDLcholesterol in the fatty fish group was also significant when compared with the lean fish group $(P=0.033)$. No differences were observed between the groups regarding within-groups differences for serum concentrations of NEFA, total cholesterol, LDL-cholesterol and total bile acids after 4 weeks.

\section{Glucose homoeostasis}

A comparison of 4 weeks of intake of lean fish, fatty fish and lean meat showed no effects on fasting concentrations of glucose, insulin, insulin C-peptide and fructosamine in either group (Table 5). We observed no differences between the groups for changes from fasting to postprandial serum concentrations of glucose, insulin and insulin C-peptide after 4 weeks. No differences were observed between the groups regarding the within-group changes in circulating concentrations of MG, CML, CRP and adiponectin.

\section{Discussion}

Numerous studies in overweight, dyslipidaemic, hypertensive, insulin-resistant and/or diabetic subjects have been conducted to investigate the effects of fish intake on serum lipids and glucose regulation ${ }^{(12,13,17,18,29-35,47)}$; however, no studies on the effect of high fish intake in a group of young, healthy, nonsmoking, normal-weight adults have been published to date. In the present study, we investigated the effects of high intake of lean or fatty fish compared with lean meat for 4 weeks on serum lipids and glucose regulation in healthy, normal-weight adults. The results from this study suggest that a high fatty fish intake for 4 weeks as part of the participants' normal diet was sufficient to affect fasting serum concentrations of TAG and HDL-cholesterol in young, normal-weight adults, but fatty fish intake did not affect glucose regulation. The observed effects on 
Table 4. Fasting concentrations of lipids, NEFA and bile acids in serum ${ }^{\star}$ (Medians and 25th-75th percentiles)

\begin{tabular}{|c|c|c|c|c|c|c|c|}
\hline & \multicolumn{2}{|c|}{ Baseline } & \multicolumn{2}{|c|}{4 weeks } & \multirow[b]{2}{*}{$P+$} & \multirow[b]{2}{*}{$P \neq$} & \multirow[b]{2}{*}{$P \S$} \\
\hline & Median & 25th-75th percentile & Median & 25th-75th percentile & & & \\
\hline \multicolumn{8}{|l|}{ TAG $(\mathrm{mmol} / \mathrm{l})$} \\
\hline Lean fish & 0.61 & $0.46,0.83$ & 0.64 & $0.43,0.80$ & 0.86 & \multirow[t]{3}{*}{0.040} & $0.33^{A}$ \\
\hline Fatty fish & 0.79 & $0.69,1.15$ & 0.73 & $0.55,0.84$ & 0.012 & & $0.018^{\mathrm{B}}$ \\
\hline Lean meat & 0.73 & $0.65,0.84$ & 0.66 & $0.50,1.26$ & 0.33 & & $0.085^{\mathrm{C}}$ \\
\hline \multicolumn{8}{|l|}{ NEFA (mmol/l) } \\
\hline Lean fish & 0.47 & $0.35,0.53$ & 0.31 & $0.22,0.43$ & 0.0060 & \multirow[t]{3}{*}{0.95} & \\
\hline Fatty fish & 0.45 & $0.32,0.57$ & 0.34 & $0.22,0.45$ & 0.046 & & \\
\hline Lean meat & 0.42 & $0.28,0.52$ & 0.26 & $0.17,0.36$ & 0.051 & & \\
\hline \multicolumn{8}{|c|}{ Total cholesterol $(\mathrm{mmol} / \mathrm{l})$} \\
\hline Lean fish & 4.6 & $4 \cdot 1,4.9$ & 4.7 & $3.9,4 \cdot 8$ & 0.53 & \multirow{3}{*}{0.86} & \\
\hline Fatty fish & 4.5 & $3.9,4.8$ & 4.4 & $3.9,4.9$ & 0.94 & & \\
\hline Lean meat & 4.8 & $3.9,5.5$ & $5 \cdot 0$ & $3 \cdot 5,5 \cdot 4$ & 0.57 & & \\
\hline \multicolumn{8}{|c|}{ HDL-cholesterol (mmol/l) } \\
\hline Lean fish & 1.7 & $1 \cdot 4,2 \cdot 0$ & 1.6 & $1.4,1.9$ & 0.28 & \multirow[t]{3}{*}{0.032} & $0.96^{\mathrm{A}}$ \\
\hline Fatty fish & 1.6 & $1.4,1.8$ & 1.7 & $1 \cdot 4,2 \cdot 0$ & 0.034 & & $0.025^{\mathrm{B}}$ \\
\hline Lean meat & 1.5 & $1 \cdot 4,2 \cdot 2$ & 1.4 & $1 \cdot 3,2 \cdot 1$ & 0.23 & & $0.033^{C}$ \\
\hline \multicolumn{8}{|c|}{ LDL-cholesterol (mmol/l) } \\
\hline Lean fish & 2.6 & $2 \cdot 3,3 \cdot 0$ & $2 \cdot 6$ & $2 \cdot 1,3.9$ & 1.0 & \multirow[t]{3}{*}{0.95} & \\
\hline Fatty fish & $2 \cdot 4$ & $2 \cdot 0,2 \cdot 8$ & $2 \cdot 4$ & $2 \cdot 0,2 \cdot 7$ & 0.97 & & \\
\hline Lean meat & 2.5 & $2 \cdot 0,3 \cdot 1$ & $2 \cdot 7$ & $1 \cdot 9,3 \cdot 2$ & 0.68 & & \\
\hline \multicolumn{8}{|c|}{ Total bile acids $(\mu \mathrm{mol} / \mathrm{l})$} \\
\hline Lean fish & 1.0 & $0.9,3.0$ & $2 \cdot 0$ & $1.5,3.0$ & 0.28 & \multirow[t]{3}{*}{0.48} & \\
\hline Fatty fish & 1.0 & $0.7,2.3$ & $2 \cdot 0$ & $1 \cdot 0,4 \cdot 3$ & 0.045 & & \\
\hline Lean meat & 1.0 & $0.9,2.0$ & 3.0 & $2 \cdot 0,5 \cdot 0$ & 0.082 & & \\
\hline
\end{tabular}

${ }^{\star}$ No differences were observed between the groups at baseline (Kruskal-Wallis test). Results are presented for thirteen participants in the lean fish group, fourteen participants in the fatty fish group and eleven participants in the lean meat group.

$\dagger$ Within-group changes are tested using Wilcoxon's signed-rank test.

¥ Changes within lean fish, fatty fish and lean meat groups are compared using the Kruskal-Wallis test.

$\S$ Changes within the lean fish group are compared with the lean meat group (A), changes within the fatty fish group are compared with the lean meat group (B), changes within the lean fish group are compared with the fatty fish group (C) using the Mann-Whitney test when the Kruskal-Wallis test showed differences between the groups.

serum lipids seen after high fatty fish intake could neither be explained by weight loss, as body weight was not affected in the fatty fish group, nor could it be explained by change in energy or macronutrient intakes or the choices of preparation of dinner meals and side dishes. No effects were observed on serum lipids and glucose regulation after lean fish intake when compared with lean meat intake.

It has been hypothesised by others that the choice of preparation methods of fish and meat and the choices of side dishes and accessories may explain at least some of the different health effects observed after fish intake when compared with meat ${ }^{(38-41)}$. Therefore, in the present study, it was mandatory for the participants to follow certain recipes with similar preparation methods and side dishes for dinner meals with lean fish, fatty fish and lean meat. In general, the contents of protein and carbohydrates were similar for meals containing fish or meat for each recipe, whereas the fat contents, and thus the energy contents, were always higher in the corresponding meals containing fatty fish. Thus, the intake of fats from dinner meals, especially of MUFA and PUFA, was higher in the fatty fish group. The participants could choose freely between the recipes for preparation of study dinners, and no differences were found between the groups in preferences for the different recipes and side dishes; thus, the current findings regarding serum lipid can probably not be explained by differences in preparation methods or dinner accessories. Despite the higher content of energy and fat in the dinners of the fatty fish group, no differences were observed in intake of energy and fat from baseline to 4 weeks in this group, suggesting that the participants regulated their intake of energy from other meals to maintain a constant intake during the study period.

The beneficial effects of fish (especially fatty fish) and fish oil intake on lipid concentrations have been reported by others $^{(12,13,29-35)}$. In the present study, in healthy, non-smoking subjects with normal BMI, high intake of fatty fish for 4 weeks resulted in reduced serum TAG and elevated HDL-cholesterol concentrations when compared with high intake of lean meat and higher HDL-cholesterol concentration when compared with lean fish intake. Our results on fatty fish intake resemble reports on $n-3$ PUFA supplementation showing decreased TAG and increased HDL-cholesterol concentrations in the circulation $^{(6,7,11-14)}$. Thus, the observed effects in the fatty fish group may be caused by the high intake of $n$ - 3 PUFA, especially as these changes were not induced by lean fish intake. It should be noted that as all participants had serum lipid concentrations within the normal reference ranges the clinical significance of the effects of fatty fish intake on TAG and HDL-cholesterol concentrations in serum is uncertain.

We have recently shown that cod protein supplementation reduced serum LDL-cholesterol concentration in overweight adults, with no effect on serum concentrations of TAG, total cholesterol and HDL-cholesterol ${ }^{(17)}$. Reduced serum TAG has been reported in overweight subjects when lean fish was part of 
Table 5. Serum concentrations of glucose, insulin, insulin C-peptide, fructosamine, methylglyoxal (MG), $N^{E}$-(carboxymethyl) lysine (CML), C-reactive protein (CRP) and adiponectin*

(Medians and 25th-75th percentiles)

\begin{tabular}{|c|c|c|c|c|c|c|}
\hline & \multicolumn{2}{|c|}{ Baseline } & \multicolumn{2}{|c|}{4 weeks } & \multirow[b]{2}{*}{$P \dagger$} & \multirow[b]{2}{*}{$P \ddagger$} \\
\hline & Median & 25th-75th percentile & Median & 25th-75th percentile & & \\
\hline \multicolumn{7}{|c|}{ Fasting glucose $(\mathrm{mmol} / \mathrm{l})$} \\
\hline Lean fish & 4.6 & $4 \cdot 4,4 \cdot 8$ & 4.6 & $4 \cdot 5,5 \cdot 0$ & 0.45 & \multirow[t]{3}{*}{0.29} \\
\hline Fatty fish & 4.9 & $4 \cdot 7,5 \cdot 0$ & 4.9 & $4 \cdot 5,5 \cdot 0$ & 0.35 & \\
\hline Lean meat & $4 \cdot 8$ & $4 \cdot 6,5 \cdot 2$ & $5 \cdot 0$ & $4 \cdot 8,5 \cdot 3$ & 0.48 & \\
\hline \multicolumn{7}{|c|}{$\Delta$ Fasting to $2 \mathrm{~h}$ glucose $(\mathrm{mmol} / \mathrm{l})$} \\
\hline Lean fish & -0.3 & $-1 \cdot 0,0 \cdot 2$ & -0.5 & $-0.6,-0.3$ & 0.42 & \multirow[t]{3}{*}{0.12} \\
\hline Fatty fish & -0.9 & $-1.2,-0.5$ & -0.6 & $-0.7,-0.2$ & 0.023 & \\
\hline Lean meat & -0.8 & $-1 \cdot 3,0 \cdot 1$ & -0.9 & $-1 \cdot 1,0 \cdot 6$ & 0.45 & \\
\hline \multicolumn{7}{|c|}{ Fasting insulin $(\mathrm{pmol} / \mathrm{l})$} \\
\hline Lean fish & $16 \cdot 8$ & $13 \cdot 2,42 \cdot 6$ & $32 \cdot 4$ & $24 \cdot 6,49 \cdot 2$ & 0.033 & \multirow[t]{3}{*}{0.20} \\
\hline Fatty fish & $36 \cdot 0$ & $24 \cdot 6,60 \cdot 0$ & $29 \cdot 4$ & $25 \cdot 2,49 \cdot 2$ & 0.38 & \\
\hline Lean meat & $39 \cdot 6$ & $28 \cdot 2,75 \cdot 0$ & $56 \cdot 4$ & $30 \cdot 6,61.2$ & 0.53 & \\
\hline \multicolumn{7}{|c|}{$\Delta$ Fasting to $2 \mathrm{~h}$ insulin $(\mathrm{pmol} / \mathrm{l})$} \\
\hline Lean fish & 47.4 & $29.4,123.6$ & $40 \cdot 8$ & $26 \cdot 4,82 \cdot 8$ & 0.53 & \multirow[t]{3}{*}{0.071} \\
\hline Fatty fish & 24.6 & $5 \cdot 4,43 \cdot 2$ & $43 \cdot 2$ & $16 \cdot 2,86 \cdot 4$ & 0.011 & \\
\hline Lean meat & 23.4 & $12 \cdot 0,58 \cdot 2$ & $37 \cdot 8$ & $-7 \cdot 2,64 \cdot 8$ & 0.72 & \\
\hline \multicolumn{7}{|c|}{ Fasting insulin C-peptide (nmol/l) } \\
\hline Lean fish & 0.38 & $0.26,0.47$ & 0.46 & $0.37,0.56$ & 0.010 & \multirow[t]{3}{*}{$0 \cdot 33$} \\
\hline Fatty fish & 0.48 & $0.39,0.53$ & 0.44 & $0.38,0.54$ & 0.82 & \\
\hline Lean meat & 0.55 & $0.35,0.63$ & 0.50 & $0.40,0.62$ & 0.48 & \\
\hline \multicolumn{7}{|c|}{$\Delta$ Fasting to $2 \mathrm{~h}$ insulin C-peptide $(\mathrm{nmol} / \mathrm{l})$} \\
\hline Lean fish & 0.33 & $0.25,0.92$ & 0.44 & $0.22,0.61$ & 0.86 & \multirow[t]{3}{*}{0.46} \\
\hline Fatty fish & 0.27 & $0.13,0.46$ & 0.33 & $0.14,0.59$ & 0.11 & \\
\hline Lean meat & 0.22 & $0.11,0.51$ & 0.20 & $0.13,0.44$ & 0.93 & \\
\hline \multicolumn{7}{|c|}{ Fasting glucose:insulin ratio (mmol glucose/pmol insulin) } \\
\hline Lean fish & 0.26 & $0.11,0.34$ & 0.14 & $0.11,0.20$ & 0.016 & \multirow[t]{3}{*}{0.25} \\
\hline Fatty fish & 0.13 & $0.08,0.19$ & 0.17 & $0.10,0.20$ & 0.68 & \\
\hline Lean meat & 0.13 & $0.06,0.17$ & 0.09 & $0.08,0.17$ & 0.59 & \\
\hline \multicolumn{7}{|c|}{ Fasting fructosamine $(\mu \mathrm{mol} / \mathrm{l})$} \\
\hline Lean fish & 252 & 247,261 & 249 & 238,257 & 0.011 & \multirow[t]{3}{*}{0.19} \\
\hline Fatty fish & 251 & 233,260 & 244 & 236,258 & 0.78 & \\
\hline Lean meat & 249 & 245,257 & 246 & 240,258 & 0.35 & \\
\hline \multicolumn{7}{|c|}{ Fasting $M G(\mathrm{ng} / \mathrm{ml})$} \\
\hline Lean fish & 172 & 132, 195 & 170 & 148,194 & 0.17 & 0.79 \\
\hline Fatty fish & 185 & 169,200 & 185 & 172,203 & 0.27 & \\
\hline Lean meat & 190 & 183,209 & 191 & 181,212 & 0.66 & \\
\hline Fasting CML & & & & & & \\
\hline Lean fish & 4.6 & $1 \cdot 9,6 \cdot 2$ & 4.7 & $2 \cdot 5,5 \cdot 8$ & 0.92 & 0.93 \\
\hline Fatty fish & 4.4 & $3 \cdot 3,5 \cdot 9$ & 4.6 & $4 \cdot 0,5 \cdot 7$ & 0.73 & \\
\hline Lean meat & 5.9 & $3 \cdot 0,7 \cdot 1$ & 6.4 & $2 \cdot 8,7 \cdot 1$ & 0.72 & \\
\hline CRP (mg/l) & & & & & & \\
\hline Lean fish & 0.6 & $0.3,0.9$ & 0.4 & $0.3,1.0$ & 0.96 & 0.97 \\
\hline Fatty fish & 0.3 & $0.7,0.8$ & 0.4 & $0.2,0.9$ & 0.75 & \\
\hline Lean meat & 0.5 & $0.2,1.0$ & 0.4 & $0.3,0.6$ & 0.88 & \\
\hline Fasting adipor & (ml) & & & & & \\
\hline Lean fish & 18.5 & $10 \cdot 5,35 \cdot 1$ & 23.3 & $10 \cdot 4,39.6$ & 0.075 & 0.73 \\
\hline Fatty fish & 17.9 & $10 \cdot 4,23.9$ & $21 \cdot 1$ & $11 \cdot 6,35.5$ & 0.041 & \\
\hline Lean meat & 19.9 & $16 \cdot 7,29 \cdot 3$ & 23.3 & $15 \cdot 1,32 \cdot 3$ & 0.72 & \\
\hline
\end{tabular}

* No differences were observed between the groups at baseline (Kruskal-Wallis test). Results are presented for thirteen participants in the lean fish group, fourteen participants in the fatty fish group and eleven participants in the lean meat group.

$\dagger$ Within-group changes are tested using the Wilcoxon's signed-rank test.

$\ddagger$ Changes within lean fish, fatty fish and lean meat groups are compared using the Kruskal-Wallis test.

an energy-restricted $\operatorname{diet}^{(32)}$, whereas others found no effect of lean fish intake on serum lipids in overweight, insulin-resistant subjects $^{(47)}$. In the present study, in subjects with normal BMI, however, lean fish intake did not affect serum TAG and cholesterol concentrations.

Intake of lean meat did not affect serum TAG and cholesterol concentrations in our study, which is in line with findings by others in overweight adults ${ }^{(36)}$ or patients with type 2 diabetes $^{(37)}$ after intake of lean meat.
There is controversy about the glucose-regulating effects of fatty fish or fish oil ${ }^{(9-11,31,35,48)}$; however, studies in rats that are obese and/or fed high-fat or high-carbohydrate diets suggest that intake of fish proteins may improve glucose tolerance ${ }^{(21-24,26)}$. Clinical studies also suggest that fish proteins may improve glucose regulation in overweight/obese participants ${ }^{(17-18)}$. In the present study, there were no changes in fasting glucose, insulin or insulin C-peptide after either fish or meat intake. Postprandial regulation of glucose may be of more 
interest than solely looking at fasting concentrations of glucose, insulin and insulin C-peptide, as humans spend most of their time in a postprandial state. In our healthy participants, the 2-h glucose concentration was lower than fasting concentrations for almost all participants, whereas the concentrations of insulin and insulin C-peptide increased postprandially. We found no differences between the within-group changes after intakes of lean fish, fatty fish or lean meat regarding fasting concentrations of glucose, insulin, insulin C-peptide, adiponectin and fructosamine, or the fasting glucose:insulin ratio. In addition, the relative increase in concentrations of glucose, insulin and insulin C-peptide from fasting to 2-h concentrations after the standardised breakfast were similar in all groups, strongly suggesting that glucose tolerance was not affected by lean or fatty fish intake. In addition, the unchanged plasma concentrations of MG and CML after fish intake suggest no change in production of advanced glycation end products.

The lack of effect of lean fish intake in the present study is in contrast to our previous study, showing that an intake of $6 \mathrm{~g}$ of cod protein as supplement (corresponding to approximately $30 \mathrm{~g}$ cod fillet) was sufficient to affect postprandial glucose and insulin concentrations in overweight subjects ${ }^{(17)}$. The discrepancy in findings between the present population with normal BMI and the overweight population ${ }^{(17)}$ may be caused by differences in the participants' regulation of glucose metabolism, as glucose regulation was most likely impaired in the overweight subjects.

Elevated circulating NEFA concentration is regarded as a risk factor for the development of insulin resistance in humans ${ }^{(49)}$. In the present study, serum NEFA was significantly reduced after 4 weeks of intake of lean fish or fatty fish; however, no differences were observed between fish and meat groups when the within-group changes were compared. Little is known about how fish and meat intakes affect circulating NEFA concentrations in humans, and future studies are warranted in populations with increased risk of developing insulin resistance and type 2 diabetes.

In the present study, participants were healthy, normal weight subjects without elevated CRP at baseline, and no changes in CRP concentration were observed in any intervention group. The literature on the effects of fish intake on CRP as a marker of inflammation is not consistent, as some studies report no effect of intake of fish on CRP in overweight men ${ }^{(33)}$ and non-obese subjects ${ }^{(35)}$, whereas others report an inverse relationship between serum CRP and fish intake in both healthy and insulin-resistant adults ${ }^{(8,47,50)}$.

There are some limitations to this study. Participants were given five different recipes to prepare lean fish-, fatty fish- or lean meat-based dinner meals, and they were encouraged to vary between the different recipes. Although great efforts were made to design menus with similar vegetables and other accessories, some differences may have occurred. However, we believe that this would not affect the outcome of the study as the preferences for preparation methods and choice of side dishes and accessories were not different between the groups. Moreover, the groups had similar intakes of energy and macronutrients during the course of the study, which may strengthen the interpretation that high intake of fatty fish caused the observed effects on serum TAG and HDL-cholesterol. As no physiological marker of the compliance was used in this study, participants were interviewed about their intake during the intervention period. More studies are needed to achieve generalisability of our findings. In the present study, the participants were students at the Faculty of Medicine and Dentistry and possibly were more health and nutrition conscious than the general population of the same age. We consider the present study to be a pilot study that is hypothesis generating rather than hypothesis testing, as there is no information available about the necessary sample size; the study will constitute a base for sample size calculations for future studies with similar designs, and no adjustment for multiple testing has been performed. However, other studies of fish intake in other populations have shown that a sample size of between ten and fifteen was sufficient to observe a lowering effect of fish intake on serum TAG concentration ${ }^{(29,31,33)}$.

\section{Conclusions}

The hypothesis of this study was that a high intake of lean or fatty fish would not affect circulating concentrations of lipids or glucose tolerance in healthy, normal-weight adults with serum concentrations of lipids, glucose and insulin within normal reference ranges, when compared with lean meat intake. Contrary to our hypothesis, our findings suggests that a high intake of fatty fish reduced serum TAG and increased HDL-cholesterol concentrations when compared with lean meat intake. In addition, fatty fish intake increased HDLcholesterol when compared with lean fish intake in this population. Our findings may possibly be due to the high content of long-chain $n-3$ PUFA in fatty fish. This is of particular interest as an improvement in lipid concentrations after fish intake has mainly been explained by others as a result of weight loss when fish was included in an energy-restricted diet $^{(32,51)}$; however, there was no change in body weight in the present study. In addition, there was no change in the intakes of energy or macronutrients within any of the experimental groups or between the groups. The observed changes in lipids in the fatty fish group could not be explained by different preparation methods or side dishes for the dinner meals.

\section{Acknowledgements}

The kind contributions of chicken and fish for the intervention trial by ICA Maxi (Bergen, Norway) and Lerøy Seafood Group ASA (Bergen, Norway) are highly appreciated. The authors thank all participants who have contributed to the present study.

The present research has been supported by funding from the Bergen Medical Research Foundation and KG Jebsen Center for Diabetes Research.

G. R., H. S., G. M. and O. A. G. designed the study. I. V. H., A. H., M. B. and O. A. G. conducted the study. I. V. H., A. H., M. B., K. A. B. and O. A. G. analysed the data and performed statistical analyses. O. A. G. drafted the paper and had primary responsibility for the final content. All authors have contributed to the writing and approved the final version of the manuscript. 
G. R. and H. S. are employed in Skretting Aquaculture Research Centre AS and Lerøy Seafood Group ASA, respectively. Skretting Aquaculture Research Centre AS is a global leader in providing innovative and sustainable nutritional solutions for the aquaculture industry. Lerøy Seafood Group ASA is the leading exporter of seafood from Norway and the world's second largest producer of Atlantic Salmon. Skretting Aquaculture Research Centre AS and Lerøy Seafood Group ASA were not involved in on-site data collection. I. V. H., A. H., M. B., K. A. B., G. M. and O. A. G. declare no conflicts of interest.

\section{Supplementary material}

For supplementary material/s referred to in this article, please visit http://dx.doi.org/doi:10.1017/S0007114516002555

\section{References}

1. Feskens EJ, Bowles CH \& Kromhout D (1991) Inverse association between fish intake and risk of glucose intolerance in normoglycemic elderly men and women. Diabetes Care 14, 935-941.

2. Whelton SP, He J, Whelton PK, et al. (2004) Meta-analysis of observational studies on fish intake and coronary heart disease. Am J Cardiol 93, 1119-1123.

3. Patel PS, Forouhi NG, Kuijsten A, et al. (2012) The prospective association between total and type of fish intake and type 2 diabetes in 8 European countries: EPIC-InterAct Study. Am J Clin Nutr 95, 1445-1453.

4. Nkondjock A \& Receveur O (2003) Fish-seafood consumption, obesity, and risk of type 2 diabetes: an ecological study. Diabetes Metab 29, 635-642.

5. Kromhout D, Bosschieter EB \& de Lezenne Coulander C (1985) The inverse relation between fish consumption and 20-year mortality from coronary heart disease. $N$ Engl J Med 312, 1205-1209.

6. Harris WS (1989) Fish oils and plasma lipid and lipoprotein metabolism in humans: a critical review. J Lipid Res 30, 785-807.

7. Harris WS (1997) n-3 Fatty acids and serum lipoproteins: human studies. Am J Clin Nutr 65, 1645S-1654S.

8. Smith KM, Barraj LM, Kantor M, et al. (2009) Relationship between fish intake, $n-3$ fatty acids, mercury and risk markers of CHD (National Health and Nutrition Examination Survey 1999-2002). Public Health Nutr 12, 1261-1269.

9. Kromhout D \& de Goede J (2014) Update on cardiometabolic health effects of omega-3 fatty acids. Curr Opin Lipidol 25, $85-90$.

10. Glauber H, Wallace P, Griver K, et al. (1988) Adverse metabolic effect of omega-3 fatty acids in non-insulindependent diabetes mellitus. Ann Intern Med 108, 663-668.

11. Popp-Snijders C, Schouten JA, Heine RJ, et al. (1987) Dietary supplementation of omega-3 polyunsaturated fatty acids improves insulin sensitivity in non-insulin-dependent diabetes. Diabetes Res $\mathbf{4}, 141-147$.

12. Eslick GD, Howe PR, Smith C, et al. (2009) Benefits of fish oil supplementation in hyperlipidemia: a systematic review and meta-analysis. Int J Cardiol 136, 4-16.

13. Nettleton JA \& Katz R (2005) $n$-3 Long-chain polyunsaturated fatty acids in type 2 diabetes: a review. J Am Diet Assoc 105, 428-440.

14. Balk EM, Lichtenstein AH, Chung M, et al. (2006) Effects of omega-3 fatty acids on serum markers of cardiovascular disease risk: a systematic review. Atherosclerosis 189, 19-30.

15. Jacques H (1990) Effects of dietary fish protein on plasma cholesterol and lipoproteins in animal models and in humans. Monogr Atheroscler 16, 59-70.
16. Thorsdottir I, Tomasson H, Gunnarsdottir I, et al. (2007) Randomized trial of weight-loss-diets for young adults varying in fish and fish oil content. Int J Obes (Lond) 31, 1560-1566.

17. Vikoren LA, Nygard OK, Lied E, et al. (2013) A randomised study on the effects of fish protein supplement on glucose tolerance, lipids and body composition in overweight adults. Br J Nutr 109, 648-657.

18. Ouellet V, Marois J, Weisnagel SJ, et al. (2007) Dietary cod protein improves insulin sensitivity in insulin-resistant men and women: a randomized controlled trial. Diabetes Care $\mathbf{3 0}$, 2816-2821.

19. Wergedahl H, Liaset B, Gudbrandsen OA, et al. (2004) Fish protein hydrolysate reduces plasma total cholesterol, increases the proportion of HDL cholesterol, and lowers Acyl-CoA: cholesterol acyltransferase activity in liver of zucker rats. J Nutr 134, 1320-1327.

20. Hosomi R, Fukunaga K, Arai H, et al. (2011) Fish protein decreases serum cholesterol in rats by inhibition of cholesterol and bile acid absorption. J Food Sci 76, H116-H121.

21. Lavigne C, Marette A \& Jacques H (2000) Cod and soy proteins compared with casein improve glucose tolerance and insulin sensitivity in rats. Am J Physiol Endocrinol Metab 278, E491-E500.

22. Pilon G, Ruzzin J, Rioux LE, et al. (2011) Differential effects of various fish proteins in altering body weight, adiposity, inflammatory status, and insulin sensitivity in high-fat-fed rats. Metabolism 60, 1122-1130.

23. Madani Z, Louchami K, Sener A, et al. (2012) Dietary sardine protein lowers insulin resistance, leptin and TNF-alpha and beneficially affects adipose tissue oxidative stress in rats with fructose-induced metabolic syndrome. Int J Mol Med 29, 311-318.

24. Lavigne C, Tremblay F, Asselin G, et al. (2001) Prevention of skeletal muscle insulin resistance by dietary cod protein in high fat-fed rats. Am J Physiol Endocrinol Metab 281, E62-E71.

25. Shukla A, Bettzieche A, Hirche F, et al. (2006) Dietary fish protein alters blood lipid concentrations and hepatic genes involved in cholesterol homeostasis in the rat model. Br J Nutr 96, 674-682

26. Drotningsvik A, Mjos SA, Hogoy I, et al. (2015) A low dietary intake of cod protein is sufficient to increase growth, improve serum and tissue fatty acid compositions, and lower serum postprandial glucose and fasting non-esterified fatty acid concentrations in obese Zucker fa/fa rats. Eur J Nutr $\mathbf{5 4}$, $1151-1160$

27. Glikmanas G, Sarmini H, Bigorie B, et al. (1988) Evaluation of Roche fructosamine test: use for diabetic patient monitoring. Clin Biochem 21, 319-321.

28. Singh R, Barden A, Mori T, et al. (2001) Advanced glycation end-products: a review. Diabetologia 44, 129-146.

29. Telle-Hansen VH, Larsen LN, Hostmark AT, et al. (2012) Daily intake of cod or salmon for 2 weeks decreases the 18:1n-9/ 18:0 ratio and serum triacylglycerols in healthy subjects. Lipids 47, 151-160

30. Schmidt EB, Skou HA, Christensen JH, et al. (2000) n-3 Fatty acids from fish and coronary artery disease: implications for public health. Public Health Nutr 3, 91-98.

31. Mori TA, Bao DQ, Burke V, et al. (1999) Dietary fish as a major component of a weight-loss diet: effect on serum lipids, glucose, and insulin metabolism in overweight hypertensive subjects. Am J Clin Nutr 70, 817-825.

32. Gunnarsdottir I, Tomasson H, Kiely M, et al. (2008) Inclusion of fish or fish oil in weight-loss diets for young adults: effects on blood lipids. Int J Obes (Lond) 32, 1105-1112.

33. Lindqvist HM, Langkilde AM, Undeland I, et al. (2009) Herring (Clupea harengus) intake influences lipoproteins but not 
inflammatory and oxidation markers in overweight men. $\mathrm{BrJ}$ Nutr 101, 383-390.

34. Tinker LF, Parks EJ, Behr SR, et al. (1999) (n-3) Fatty acid supplementation in moderately hypertriglyceridemic adults changes postprandial lipid and apolipoprotein B responses to a standardized test meal. J Nutr 129, 1126-1134.

35. Lara JJ, Economou M, Wallace AM, et al. (2007) Benefits of salmon eating on traditional and novel vascular risk factors in young, non-obese healthy subjects. Atherosclerosis $\mathbf{1 9 3}$, 213-221

36. Murphy KJ, Thomson RL, Coates AM, et al. (2012) Effects of eating fresh lean pork on cardiometabolic health parameters. Nutrients 4, 711-723.

37. Gross JL, Zelmanovitz T, Moulin CC, et al. (2002) Effect of a chicken-based diet on renal function and lipid profile in patients with type 2 diabetes: a randomized crossover trial. Diabetes Care 25, 645-651.

38. Mozaffarian D, Lemaitre RN, Kuller LH, et al. (2003) Cardiac benefits of fish consumption may depend on the type of fish meal consumed: the Cardiovascular Health Study. Circulation 107, 1372-1377.

39. Stephen NM, Jeya Shakila R, Jeyasekaran G, et al. (2010) Effect of different types of heat processing on chemical changes in tuna. J Food Sci Technol 47, 174-181.

40. Mnari Bhouri A, Jrah Harzallah H, Dhibi M, et al. (2010) Nutritional fatty acid quality of raw and cooked farmed and wild sea bream (Sparus aurata). J Agric Food Chem 58, 507-512.

41. Larsen R, Stormo SK, Dragnes BT, et al. (2007) Losses of taurine, creatine, glycine and alanine from cod (Gadus morhua L.) fillet during processing. J Food Comp Anal 20, 396-402.
42. NMKL (2003) Nitrogen. Determination in Foods and Feeds According to Kjeldahl. Method No. 6, 4th ed. Oslo, Norway: NMKL.

43. NMKL (1991) Moisture and Ash. Gravimetric Determination in Meat and Meat Products. Method No. 23, 3rd ed. Oslo, Norway: NMKL.

44. Grahl-Nielsen O \& Barnung T (1985) Variations in the fatty acid profile of marine animals caused by environmental and developmental changes. Mar Environ Res 17, 218-221.

45. Lefebvre PJ \& Luyckx AS (1976) The breakfast tolerance test: a return to physiology. Diabete Metab 2, 15-19.

46. The Norwegian Food Safety Authority, the Directorate for Health and the Department of Nutrition at the University of Oslo (2010) http://matportalen.no/Emner/matpadata

47. Ouellet V, Weisnagel SJ, Marois J, et al. (2008) Dietary cod protein reduces plasma C-reactive protein in insulin-resistant men and women. J Nutr 138, 2386-2391.

48. Bhathena SJ, Berlin E, Judd JT, et al. (1991) Effects of omega 3 fatty acids and vitamin $\mathrm{E}$ on hormones involved in carbohydrate and lipid metabolism in men. Am J Clin Nutr 54, 684-688.

49. Roden M, Price TB, Perseghin G, et al. (1996) Mechanism of free fatty acid-induced insulin resistance in humans. $J$ Clin Invest 97, 2859-2865.

50. Pot GK, Geelen A, Majsak-Newman G, et al. (2010) Increased consumption of fatty and lean fish reduces serum C-reactive protein concentrations but not inflammation markers in feces and in colonic biopsies. J Nutr 140, 371-376.

51. Ramel A, Martinez JA, Kiely M, et al. (2010) Effects of weight loss and seafood consumption on inflammation parameters in young, overweight and obese European men and women during 8 weeks of energy restriction. Eur J Clin Nutr 64, 987-993. 